\title{
Absence of hole pairing in a simple $t-J$ model on the Shastry-Sutherland lattice
}

\author{
P. W. Leung* and Y. F. Cheng \\ Physics Department, Hong Kong University of Science and Technology, Clear Water Bay, Hong Kong \\ (Received 27 January 2004; revised manuscript received 14 April 2004; published 18 May 2004)
}

\begin{abstract}
The Shastry-Sutherland model is a two-dimensional frustrated spin model whose ground state is a spin gap state. We study this model doped with one and two holes on a 32-site lattice using exact diagonalization. When $t^{\prime}>0$, we find that the diagonal dimer order that exists at half-filling is retained at these moderate doping levels. No other order is found to be favored on doping. The holes are strongly repulsive unless the hopping terms are unrealistically small. Therefore, the existence of a spin gap at half-filling does not guarantee holepairing in the present case.
\end{abstract}

DOI: 10.1103/PhysRevB.69.180403

PACS number(s): 75.40.Mg, 71.10.Fd, 71.27.+a

The Shastry-Sutherland (SS) model is an exceptional example of a two-dimensional frustrated spin system that has an exact solution. ${ }^{1}$ Remarkably, it is also an excellent theoretical model for the spin gap material $\mathrm{SrCu}_{2}\left(\mathrm{BO}_{3}\right)_{2} \cdot{ }^{2}$ The SS model is a Heisenberg model with exchange interaction $J$ on a two-dimensional square lattice frustrated by diagonal bonds $J^{\prime}$ as shown in Fig. 1. When $J / J^{\prime}$ is smaller than a critical value of about $0.677,{ }^{3}$ the ground state is a direct product of orthogonal singlet dimers residing on the $J^{\prime}$ bonds. This is a spin gap state because the lowest spin excitation involves turning a singlet dimer into a triplet. Different experiments have suggested a range of $J / J^{\prime}$ for $\mathrm{SrCu}_{2}\left(\mathrm{BO}_{3}\right)_{2}$, out of which 0.635 seems to be the optimal one. A great deal of work has been devoted to studying the excited states of this model as an effort to explain various experimental results. It is found that the almost localized nature of spin excitations leads to superstructures that give rise to plateaux in the magnetization curve. Besides its importance as a theoretical model for $\mathrm{SrCu}_{2}\left(\mathrm{BO}_{3}\right)_{2}$, the $\mathrm{SS}$ model possesses a quantum phase transition. One believes that there is at least one intermediate state between the diagonal dimer state at small $J$ and the Néel state at large $J$. While the nature of the intermediate state is still controversial, it seems like the plaquette resonating-valence-bond (RVB) state and the helical state are among the most likely candidates. Although discussions on the quantum phase transition are theoretical, they are not totally irrelevant to $\mathrm{SrCu}_{2}\left(\mathrm{BO}_{3}\right)_{2}$. Since its $J / J^{\prime}$ is not too far from the critical value, it may be possible to shift it even closer by applying pressure, substitution, etc. to $\mathrm{SrCu}_{2}\left(\mathrm{BO}_{3}\right)_{2}$.

The relation between disordered spin liquids with a gap in the spin excitation and superconductivity has aroused a lot of interest. It has been suggested that doping a spin gap system may lead to hole-pairing and superconductivity. It is therefore interesting and important to identify spin gap materials that are Mott insulators with no long-range spin order. $\mathrm{SrCu}_{2}\left(\mathrm{BO}_{3}\right)_{2}$ is one such compound. Although this compound has not been doped, there is no shortage of suggestions that on doping the SS model may exhibit superconductivity. Different types of superconducting states have been suggested at different doping levels. ${ }^{4-6}$ One can draw an analogy to a doped two-leg spin ladder. ${ }^{7}$ At half-filling the ground state is a spin gap state with singlets forming on the rungs. When two holes are introduced, they tend to stay on the same rung in order to avoid breaking more singlets. This is a beautiful example of a spin gap leading to a mechanism for hole pairing. The diagonal dimer state of the SS model is also a spin gap state. But it differs from the two-leg spin ladder in that it is a two-dimensional model. It is thus interesting to see whether the same spin gap induced pairing mechanism is in effect in the SS model. Furthermore, doping may introduce more interesting physics to the spin background. When $J / J^{\prime}$ is close to but below the critical value, on doping the system may select other ordered (or disordered) states that can better support hole motion. ${ }^{8}$ Since $J / J^{\prime}$ for $\mathrm{SrCu}_{2}\left(\mathrm{BO}_{3}\right)_{2}$ is close to the critical value, theoretical discussions on the quantum phase transition may become even more relevant if it can be doped. Here we report our results on the SS model with up to two holes on a 32-site lattice using numerical diagonalization.

We start from the $t-J$ model Hamiltonian,

$$
\mathcal{H}=-\sum_{\langle i j\rangle \sigma} t_{i j}\left(\tilde{c}_{i \sigma}^{\dagger} \tilde{c}_{j \sigma}+\tilde{c}_{j \sigma}^{\dagger} \widetilde{c}_{i \sigma}\right)+\sum_{\langle i j\rangle} J_{i j}\left(\mathbf{S}_{i} \cdot \mathbf{S}_{j}-\frac{1}{4} n_{i} n_{j}\right),
$$

where $t_{i j}=t$ and $J_{i j}=J$ when $\langle i j\rangle$ is a nearest neighbor pair, and $t_{i j}=t^{\prime}$ and $J_{i j}=J^{\prime}$ when $\langle i j\rangle$ is a diagonal pair as shown in Fig. 1(a), and zero otherwise. Following Ref. 4, we define $\alpha \equiv t^{\prime} / t$. Using the large- $U$ expansion of the Hubbard model as a reference, namely $J=4 t^{2} / U$, we take $J^{\prime} / J=\alpha^{2}$. Conse-

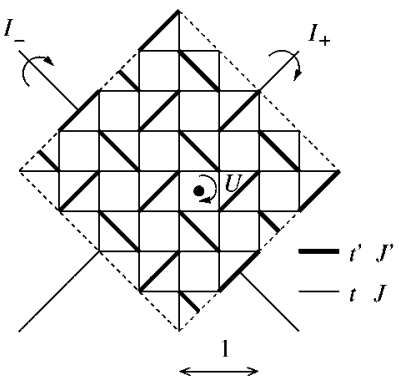

(a)

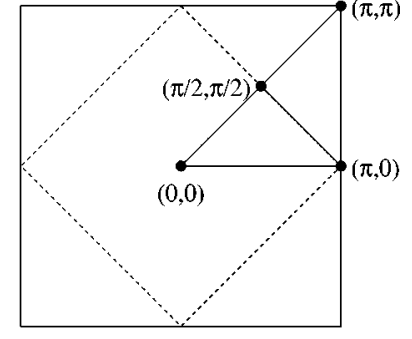

(b)
FIG. 1. (a) A 32-site Shastry-Sutherland lattice with periodic boundary conditions. Also shown are the two types of spin interactions and hole hopping terms. $I_{ \pm}$are reflections about the two axis as shown, and $U$ is a four-fold rotation about the point indicated by

- (b) The corresponding distinct reciprocal space vectors. 
TABLE I. Ground state energies and symmetries of the model with different number of holes $N_{h}$ at $\alpha=1.25$ and $J / t=0.3 . N_{B}$ is the number of basis in that particular symmetry subspace.

\begin{tabular}{cccccccc}
\hline \hline$N_{h}$ & $E / t$ & $\mathbf{k}$ & $I_{+}$ & $I_{-}$ & $U$ & $I_{\sigma}$ & $N_{B}$ \\
\hline 0 & -12.3 & $(0,0)$ & + & + & + & + & $4,708,641$ \\
1 & -15.780034 & $(0,0)$ & + & + & - & & $150,297,603$ \\
2 & -19.291015 & $(0,0)$ & + & + & + & - & $601,144,932$ \\
\hline \hline
\end{tabular}

quently there are only two parameters in our model: $\alpha$ and $J / t$. Since we want to study hole motion in a dimerized spin background and to mimic the material $\mathrm{SrCu}_{2}\left(\mathrm{BO}_{3}\right)_{2}$, we fix $\alpha=1.25$, i.e., $J / J^{\prime}=0.64$. The ground state at half-filling is the SS dimerized state with energy $-\left(J+J^{\prime}\right) N / 2$ where $N$ is the number of sites. This leaves us with only one adjustable parameter $J / t$ when holes are added. Without guidance from experimental data, we choose $J / t=0.3$, which is the appropriate value for high $T_{c}$ materials. It is also the same choice as in Ref. 4. Using exact diagonalization we solve this model on a 32-site SS lattice with periodic boundary conditions as shown in Fig. 1(a). The size of a unit cell is defined to be 1, i.e., nearest neighbor sites are at distance $1 / 2$ apart. Since there are four sites in a unit cell, an $N$-site SS lattice has N/4 translation operations. The allowed momenta are shown in Fig. 1(b). Spin reflection symmetry $I_{\sigma}$ can be used to reduce the size of the Hilbert space by a factor of 2 when the number of spins is even. In addition, the SS lattice possesses $C_{4 v}$ (or $4 \mathrm{~mm}$ ) point group symmetry. ${ }^{9}$ Therefore eigenstates can have $s, p$, or $d$ symmetries, just like the case on a square lattice. In our implementation, we choose to classify eigenstates according to their symmetry properties with respect to the two reflections $I_{+}$and the four-fold rotation $U$ as shown in Fig. 1(a). ${ }^{10}$ Table I shows the ground state properties of the model with zero, one and two holes.

The one hole ground state has $d$-like symmetry as shown in Table I. Figure 2 shows the spin structure of this ground state. It is obvious that the introduction of a mobile hole does not alter the dimerized spin background much. Most of the diagonal bonds remain as strong singlets with $\left\langle\mathbf{S}_{i} \cdot \mathbf{S}_{j}\right\rangle$ not much weaker than $-3 / 4$. The most noticeable feature is an almost free five-spin chain formed next to the hole. When a spin is removed from one dimer, the resulting free spin tends to minimize energy by forming a five-spin chain. In this way the system avoids the high energy cost of having a free spin and at the same time minimizes disruption to the spin background. Note that $\left\langle\mathbf{S}_{i} \cdot \mathbf{S}_{j}\right\rangle$ along this "chain" are not too far from the corresponding values in an isolated five-spin chain with the same $J / J^{\prime}$, which are -0.6588 and -0.2855 , respectively. Also note that it can easily lower its energy through hopping - the apex of the chain can hop to a diagonal site without disrupting the spin background. The average kinetic energy per bond on the diagonal and nearest neighbor bonds are $-0.06062 t^{\prime}$ and $-0.04597 t$, respectively, showing that diagonal hopping is more active than nearest neighbor hopping. Furthermore, this excitation is quite localized. The bandwidth is only 0.1611 , much smaller than the case on a square lattice which is about $0.6 t .^{11}$

The two-hole ground state wave function is $s$-like as

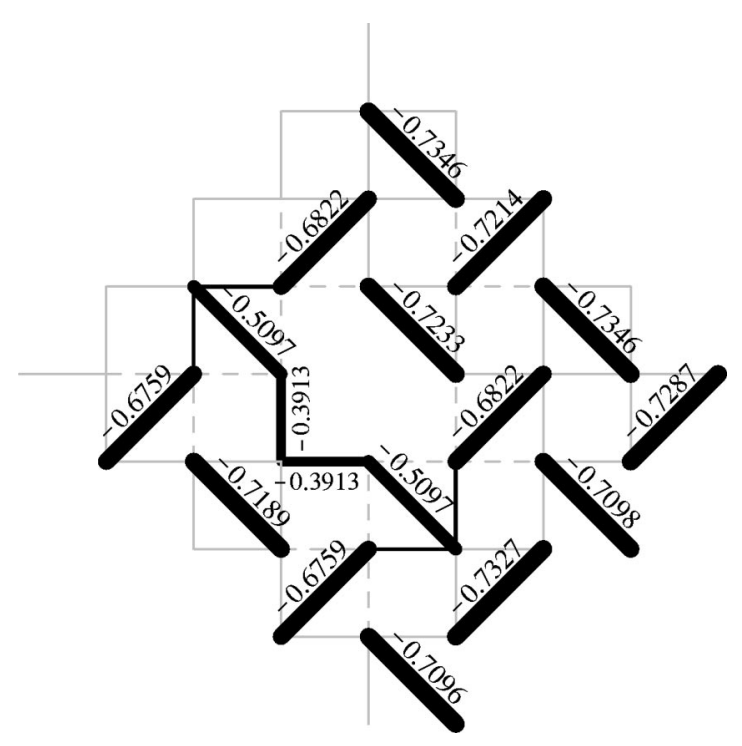

FIG. 2. Spin structure of the one-hole ground state. The hole is at the open lattice point at the center of the lattice. Thickness of the lines are proportional to the magnitudes of $\left\langle\mathbf{S}_{i} \cdot \mathbf{S}_{j}\right\rangle$, whose values are also shown for strong singlet bonds. Solid and broken lines represent negative and positive numbers, respectively. Shaded lines correspond to correlations too small to be shown in this scale. Their thickness do not represent their magnitudes, but are draw to show their signs only.

shown in Table I. The two-hole binding energy $E_{b} \equiv E_{2 h}$ $-2 E_{1 h}+E_{0}$ is $-0.0309 t$ at $J / t=0.3$. Although $E_{b}$ is negative, its small value makes it doubtful whether it really represents hole-binding. Figure 3(a) shows the hole correlation function $C_{h h}(r) \equiv\left\langle\left(1-n_{r}\right)\left(1-n_{0}\right)\right\rangle$, which is the probability of occurrence of that particular hole configuration. It clearly shows that the holes prefer to stay away from each other, i.e., they are repulsive. The configuration corresponding to hole-pairing due to spin gap effect, where the holes reside on the same diagonal bond, is indicated by " $\mathrm{A}$ " in Fig. 3 (a). Its hole correlation is the smallest. The most favorable hole configuration is when they are at maximum distance apart as indicated by "B" in Fig. 3(a). Figure 4 shows its
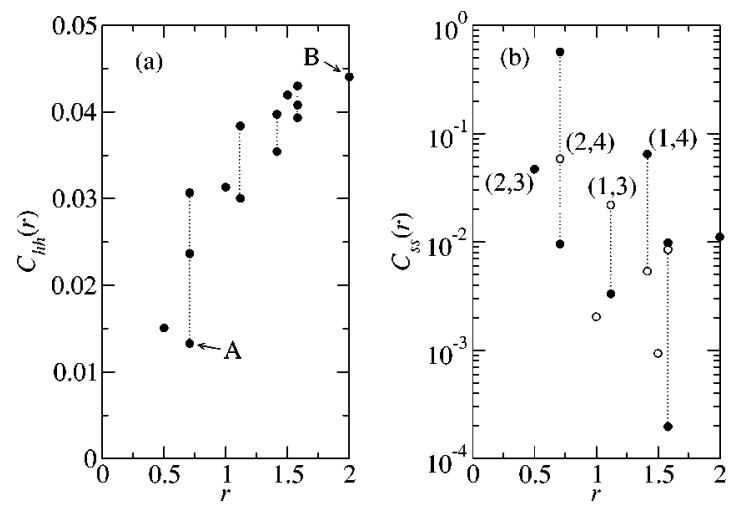

FIG. 3. (a) Hole correlation $C_{h h}(r)$ and (b) spin correlation $C_{s s}(r)$ of the two-hole model. Dotted lines join inequivalent points with the same $r$. In (b), open and closed circles represent positive and negative correlations, respectively. 


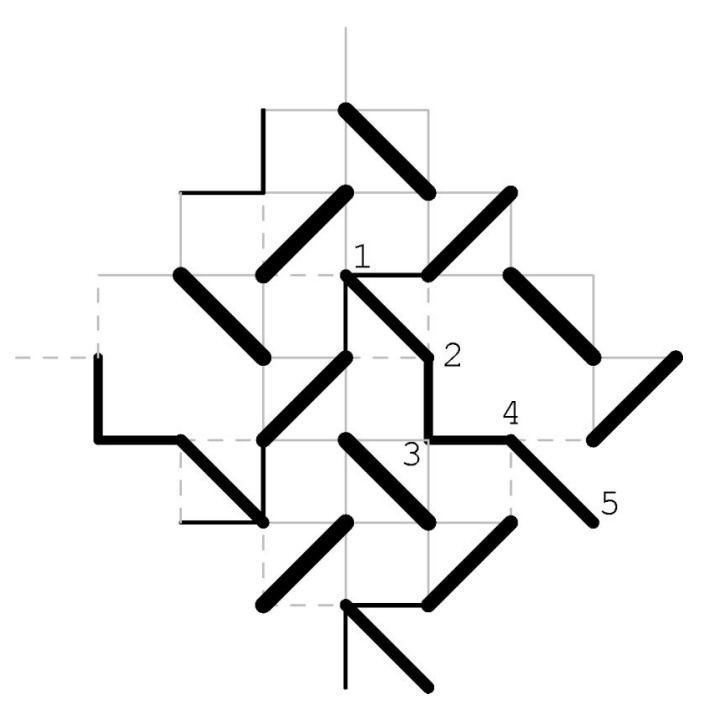

FIG. 4. Same as Fig. 2 except for the two-hole model projected to the subspace where the holes are at the two open lattice points.

spin structure. A striking feature is that next to each hole there is an almost free five-spin chain which we have seen in the one-hole ground state, Fig. 2. The spin correlation $C_{s s}(r) \equiv\left\langle\mathbf{S}_{r} \cdot \mathbf{S}_{0}\right\rangle$ shown in Fig. 3(b) is also consistent with the existence of such spin chain. In the diagonal dimer state at half-filling, $C_{s s}(r)$ is zero everywhere except when the holes are in the same diagonal bond. $C_{s s}(r)$ at this point remains the largest in Fig. 3(b), showing that dimer order persists in the two-hole ground state. In addition there are four $C_{s s}(r)$ at $r=1 / 2,1 / \sqrt{2}, \sqrt{5} / 2$, and $\sqrt{2}$ that have appreciable values. They are due to correlations between spins in the five-spin chains. For illustration purpose we label these four points in Fig. 3(b) by $(a, b)$ where $a$ and $b$ are sites on a five-spin chain such as those marked in Fig. 4. For example, the positive correlation $(2,4)$ in Fig. 3(b) is due to spins 2 and 4 in Fig. 4. Once we establish the existence of five-spin chains, we can easily understand why holes repel. If the holes are well separated such that a five-spin chain can be formed next to each hole, then the local spin energy is minimized by getting rid of the otherwise free spins, and kinetic energy is favored by the hopping motion of the apex of the spin chains.

At $t=0$ the two-hole ground state is the exact dimerized state just like at half-filling but with one dimer removed. Therefore hole pairing must exist in this limit. Let us look at how it is destroyed as $t$ increases. In Fig. 5(a) we plot the root-mean-square separation of holes $r_{\mathrm{rms}} \equiv \sqrt{\left\langle r^{2}\right\rangle}$ at different $t / J$. The horizontal broken line shows the value for two uncorrelated holes on this 32-site lattice, which is 1.1914. Let us consider the result at $\alpha=1.25$ first. At $t / J=0$, the holes are at the same diagonal bond and $r_{\mathrm{rms}}=1 / \sqrt{2}$. But as $t / J$ increases, $r_{\text {rms }}$ increases very fast and at $t / J \sim 0.1$ it already exceeds the uncorrelated value. When $t / J \gtrsim 0.2, r_{\text {rms }}$ becomes saturated and the holes become strongly repulsive. In Fig. 5(b) we plot the kinetic and spin energies which are the ground state expectation of the first and second terms of $\mathcal{H}$ in Eq. (1), respectively. Except at very small $t / J$ where $r_{\text {rms }}$ is still increasing rapidly, the kinetic energy decreases linearly
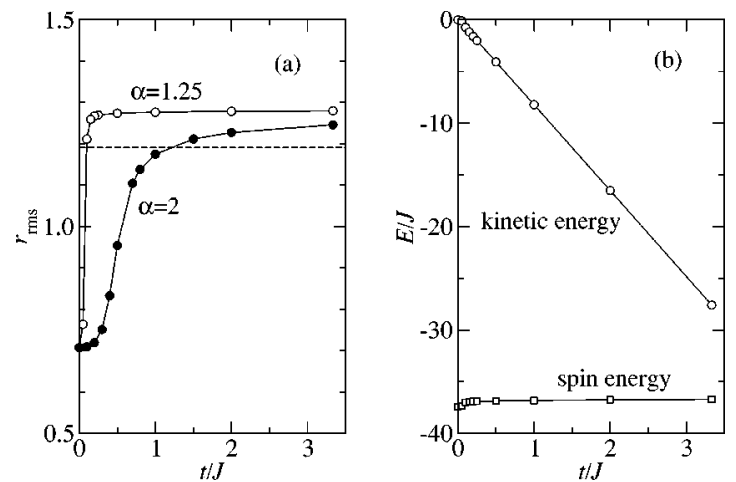

FIG. 5. (a) Root-mean-square separation of holes in the twohole ground state at $\alpha=1.25$ and 2 . The broken line indicates the value for a pair of uncorrelated holes on this 32-site lattice. (b) Kinetic and spin energies at $\alpha=1.25$.

with $t / J$ while the spin energy barely increases. This shows that once the system enters the hole-repelling regime, kinetic energy of the holes dominates and their motion do not cause much disruption to the spin background. This is in total agreement with our picture discussed above.

For the purpose of comparison we also show the corresponding result at $\alpha=2$ in Fig. 5(a). Note that this corresponds to $J / J^{\prime}=0.25$, which is far from the realistic parameter for $\mathrm{SrCu}_{2}\left(\mathrm{BO}_{3}\right)_{2}$. With a much larger spin gap - 3.7172J compared to $0.6015 J$ at $\alpha=1.25$, the resulting hole-binding effect persists to larger $t / J$, and $r_{\text {rms }}$ reaches the uncorrelated value when $t / J$ is slightly larger than 1. However, this represents only a qualitative change. The kinetic effect eventually wins and the holes are unbound when $t / J>1$.

So far our results show that diagonal dimer order is retained on doping. Next we try to look for "hidden" orders that may be associated with charge motion. Our previous study $^{12}$ has shown that the $d$-wave state of the two-hole $t$ - $J$ model on a square lattice has a staggered pattern in the current correlation $\left\langle j_{k l} j_{m n}\right\rangle$, where the current on a bond linking sites $k$ and $l$ is defined by $j_{k l}=i t_{k l}\left(\tilde{c}_{k \sigma}^{\dagger} \widetilde{c}_{l \sigma}-\tilde{c}_{l \sigma}^{\dagger} \widetilde{c}_{k \sigma}\right)$. This staggered current pattern is closely related to the staggeredflux phase based on the SU(2) theory. ${ }^{13}$ On the SS lattice, a similar staggered-flux phase has been suggested by a meanfield study. ${ }^{5}$ However, we know that on a square lattice the staggered current pattern exists only in the $d$-wave state where the holes are attractive. But in the present case the ground state has $s$, not $d$ symmetry, and the holes are repulsive. Hence we do not expect to see a staggered current pattern in this ground state. Figure 6 clearly shows no staggered pattern in the current correlation. Therefore we do not find evidence for a staggered-flux phase in this model.

Not only does dimerized order persist on doping, we believe that doping in fact stabilizes it near the quantum phase transition point. To illustrate this we repeat our calculations at $\alpha=1.2$, i.e., $J / J^{\prime}=0.694$ which is beyond the quantum phase transition point. The ground state at half-filling on a 32-site SS lattice shows plaquette order in the dimer correlation. ${ }^{14}$ However, on doping with one and two holes the system dramatically changes back to diagonal dimer or- 


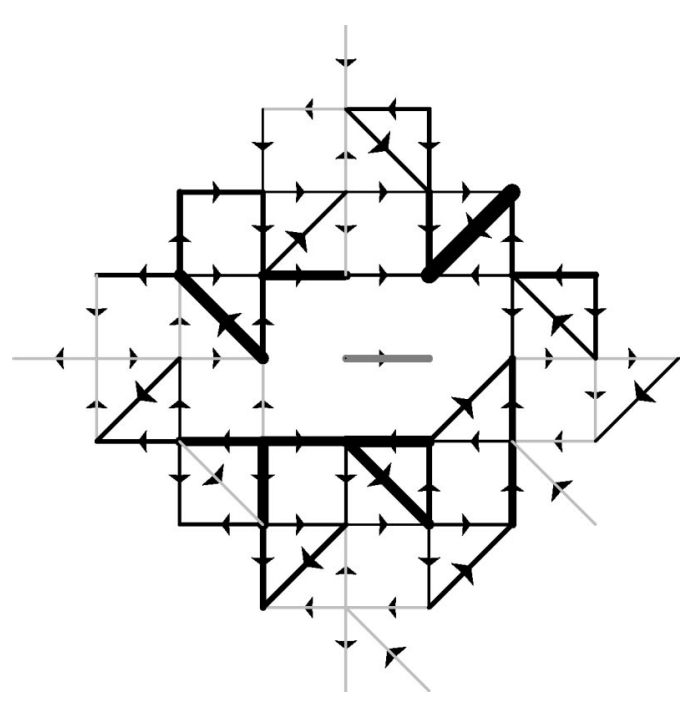

FIG. 6. The current correlation $\left\langle j_{k l} j_{m n}\right\rangle$ in the two-hole model. The reference bond is denoted by a shaded line with an arrow near the center of the diagram. Thickness of solid lines are proportional to the magnitudes of the current correlations. Thin shaded lines represent correlations that are too small to be shown in this scale.

der. The spin structures and correlations $C_{h h}(r)$ and $C_{s s}(r)$ are qualitatively the same as in Figs. 2, 4, and 3, respectively. The one- and two-hole ground state energies are $-15.214070 t$ and $-18.736734 t$, giving a binding energy $E_{b}=-0.0206 t$, compared to $-0.0309 t$ at $\alpha=1.25$. $r_{\text {rms }}$ is 1.2822 , compared to 1.2800 at $\alpha=1.25$. Since all results are so similar, our previous conclusions apply to the present case with $\alpha=1.2$, i.e., the holes are repulsive and the spin structure shows strong diagonal dimer order. Since the plaquette RVB state exists at most in a very small range of $J / J^{\prime}$, the fact that on doping it changes back to diagonal dimer order means that doping destroys plaquette RVB order and stabilizes diagonal dimer order.

To summarize, we find that doping the dimerized ground state of the SS model does not favor any other ordered or disordered state. In fact doping favors diagonal dimer order. When a mobile hole is introduced to the dimerized state, the "free spin" that would have been formed minimizes energy by forming a five-spin chain with two of its nearest dimers. This excitation is localized and has a small dispersion compared to the $t-J$ model on a square lattice. When more than one hole is present, their respective spin chains tend to avoid each other. This gives rise to short-range repulsion between the holes. Although there may exist long-range attraction between the holes mediated through the chains, Fig. 5(b) shows that the spin energy is not sensitive to changing spin configurations. Therefore we consider long-range attraction unlikely. There is another competing short-range pairing mechanism - the spin gap naturally leads to pairing of holes on the same diagonal bond when $t=0$. But we find that this pairing mechanism is in effect only when $t / J$ is smaller than about 0.2 at $\alpha=1.25$. Note that this value corresponds to $U / t \simeq 4 t / J=0.8$, which is far from the strongly interacting regime of the Hubbard model. As a result, the idea of treating the dimerized ground state of $\mathrm{SrCu}_{2}\left(\mathrm{BO}_{3}\right)_{2}$ as a Mott insulating state of an underlying Hubbard model will fail. Consequently we consider $t / J<0.2$ too small to be realistic. The implication of our result is that the existence of spin gap in a disordered spin liquid state does not guarantee charge binding.

Finally, we remark that our results do not completely rule out hole pairing in the SS model because we have studied the case $t^{\prime}>0$ only. Just like in the case of a square lattice, the sign of $t$ is irrelevant. On a square lattice, $t^{\prime}$ breaks electronhole symmetry and Néel order of the spin background is better preserved with $t^{\prime}>0$. This property is consistent with the phase diagram of electron-doped high $T_{c}$ superconductors. ${ }^{15}$ Similarly, we have shown that on a SS lattice diagonal dimer order of the spin background persists on doping when $t^{\prime}>0$. But without experimental results we cannot decide whether $t^{\prime}>0$ corresponds to electron-doping in the present case. Nevertheless, it is meaningful to study the case $t^{\prime}<0$ where diagonal hopping is discouraged. These works are in progress and will be presented elsewhere.

This work was supported by the Hong Kong RGC Grant No. HKUST6075/02P. Most calculations were performed on a 64-CPU AMD Opteron cluster computer in the Physics Department of HKUST.
*Electronic address: p.w.leung@ust.hk

${ }^{1}$ B.S. Shastry and B. Sutherland, Physica B \& C 108B, 1069 (1981).

${ }^{2}$ For a review see S. Miyahara and K. Ueda, J. Phys.: Condens. Matter 15, R327 (2003).

${ }^{3}$ A. Koga and N. Kawakami, Phys. Rev. Lett. 84, 4461 (2000).

${ }^{4}$ B.S. Shastry and B. Kumar, Prog. Theor. Phys. Suppl. 145, 1 (2002).

${ }^{5}$ C.H. Chung and Y.B. Kim, cond-mat/0310430 (unpublished).

${ }^{6}$ T. Kimura, K. Kuroki, R. Arita, and H. Aoki, Phys. Rev. B 69, 054501 (2004).

${ }^{7}$ For a review, see E. Dagotto and T.M. Rice, Science 271, 618 (1996).

${ }^{8}$ C.H. Chung, J.B. Marston, and S. Sachdev, Phys. Rev. B 64, 134407 (2001)
${ }^{9}$ C. Knetter, A. Bühler, E. Müller-Hartmann, and G.S. Uhrig, Phys. Rev. Lett. 85, 3958 (2000).

${ }^{10}$ T. Munehisa and Y. Munehisa, J. Phys. Soc. Jpn. 72, 160 (2003).

${ }^{11}$ P.W. Leung and R.J. Gooding, Phys. Rev. B 52, R15711 (1995). Note that on a square lattice lowest energy states at some $\mathbf{k}$ points have total spin $S>1 / 2$. They are excluded in calculating the bandwidth. But this does not happen in the present case on a SS lattice.

${ }^{12}$ P.W. Leung, Phys. Rev. B 62, R6112 (2000).

${ }^{13}$ D.A. Ivanov, P.A. Lee, and X.-G. Wen, Phys. Rev. Lett. 84, 3958 (2000).

${ }^{14}$ A. Läuchli, S. Wessel, and M. Sigrist, Phys. Rev. B 66, 014401 (2002).

${ }^{15}$ T. Tohyama and S. Maekawa, Phys. Rev. B 49, 3596 (1994); R.J. Gooding, K.J.E. Vos, and P.W. Leung, ibid. 50, 12866 (1994). 